PESQUIMAT, Revista de la F.C.M. de la

Universidad Nacional Mayor de San Marcos

Vol. XV N², pp. 39-50, Lima - Perú, Marzo 2013

\title{
PROGRAMACIÓN LINEAL APLICADA A UN TIPO DE PROGRAMACIÓN CONVEXA
}

\author{
Edinson R. Montoro Alegre ${ }^{1}$, Martha Hilda Timoteo Sanchez ${ }^{2}$, \\ Carole Huaman Oriundo ${ }^{3}$, Gladys Melgarejo Estremadoyro ${ }^{4}$
}

\begin{abstract}
Resumen: En el presente trabajo se estudia una estrategia para un tipo de problema convexo. Tratamos un problema de programación lineal cuyos coeficientes de las variables de decisión en la función objetivo tienen un comportamiento no lineal. Cuando los coeficientes son constantes el Método Simplex resuelve estos problemas sin mayor dificultad, pero cuando los coeficientes dejan de ser constantes ya el simplex no funciona. Se propone una técnica que explota el comportamiento convexo de dichos coeficientes y hace uso de la teoría de aproximación por funciones lineales a trozos.

Palabras clave: Programación convexa, funciones lineales a trozos y problema de transporte.
\end{abstract}

\section{LINEAR PROGRAMMING APPLIED TO A CONVEX PROGRAMMING TYPE}

\begin{abstract}
In the presents paper studying a strategy for a type of convex problem, we treat a linear programming problem whose coefficients of decision variables in the objective function has a nonlinear behavior. When the coefficients are constant the Simplex Method solves these problems without much difficulty, but when the coefficients are no longer constant and the Simplex does not work. We propose a technique that exploits the convex behavior of these coefficients and uses the theory of approximation by piecewise linear functions.
\end{abstract}

Keywords: Convex programming, piecewise linear functions and transport problem.

\section{Introducción}

En la mayoría de modelos de programación lineal, los costos unitarios de utilidad o de beneficio son constantes para las diferentes variables de decisión que representan algún tipo de producto.

La discusión en este trabajo se enfoca principalmente sobre el caso en el cual dichas "constantes" obedecen a un comportamiento no lineal, pero si cóncavo. Esto quiere decir que para niveles altos de producción de cierto tipo de artículo $\left(x_{i}\right)$ (o tal vez todos) que poseen un costo unitario de producción $c_{i}$ relativamente bajo, sufre un incremento. Este tipo de característica no permite una solución directa por el Método Simplex. Sin embargo, podemos deducir un método que permite una solución aproximada a aquella que es obtenida por el Método Simplex para ciertos problemas de este tipo. Vamos a considerar que la función objetivo debe ser separable con respecto a las variables de decisión $x_{i}$ y la función utilidad o beneficio total para cada una de las variables de decisión $x_{i}$ debe ser cóncava la cual debe ser maximizada.

\footnotetext{
${ }^{1}$ Universidad Nacional Mayor de San Marcos - Facultad de Ciencias Matemáticas. e-mail: edinsonmontoro@yahoo.com

${ }^{2}$ Universidad Nacional Mayor de San Marcos - Facultad de Ciencias Matemáticas. e-mail: mtimoteos@unmsm.edu.pe

${ }^{3}$ Universidad Nacional Mayor de San Marcos - Facultad de Ciencias Matemáticas e-mail:cho26_04@hotmail.com

${ }^{4}$ Universidad Nacional Mayor de San Marcos - Facultad de Ciencias Matemáticas. e-mail:ggladysme.Gyahoo.com
} 
En el caso de minimización, la función utilidad total o beneficio total para cada una de las variables de decisión debe ser separable y convexa.

\section{Ejemplo 1 (Visión general del procedimiento)}

Consideremos el problema con una sola variable que representa la cantidad de un tipo de producto a ser producido y brinda una utilidad de 2 soles por unidad producida. Asimismo se tiene una restricción de horas de trabajo por un total de 20 horas. Si se sabe que cada unidad de producto requiere una hora de trabajo, entonces podemos plantear el siguiente problema:

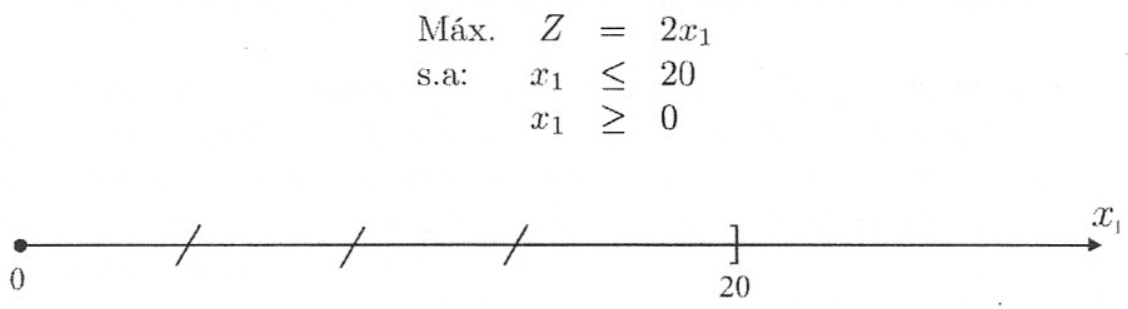

Figura 1

La solución optimal es obviamente $x_{1}=20$ y $z=40$.

Ahora, si elevamos las horas de trabajo disponible a 30 horas, la región factible será extendida a 30 sobre el eje $x_{1}$.

Sin embargo, el incremento que afecta al costo (sobre costo) por laborar a más de 20 horas es más alto, pues la utilidad que brinda cada unidad del producto $x_{1}$ superior a las 20 horas brinda una utilidad de 1 sol. Es decir, la función de utilidad total no es lineal.

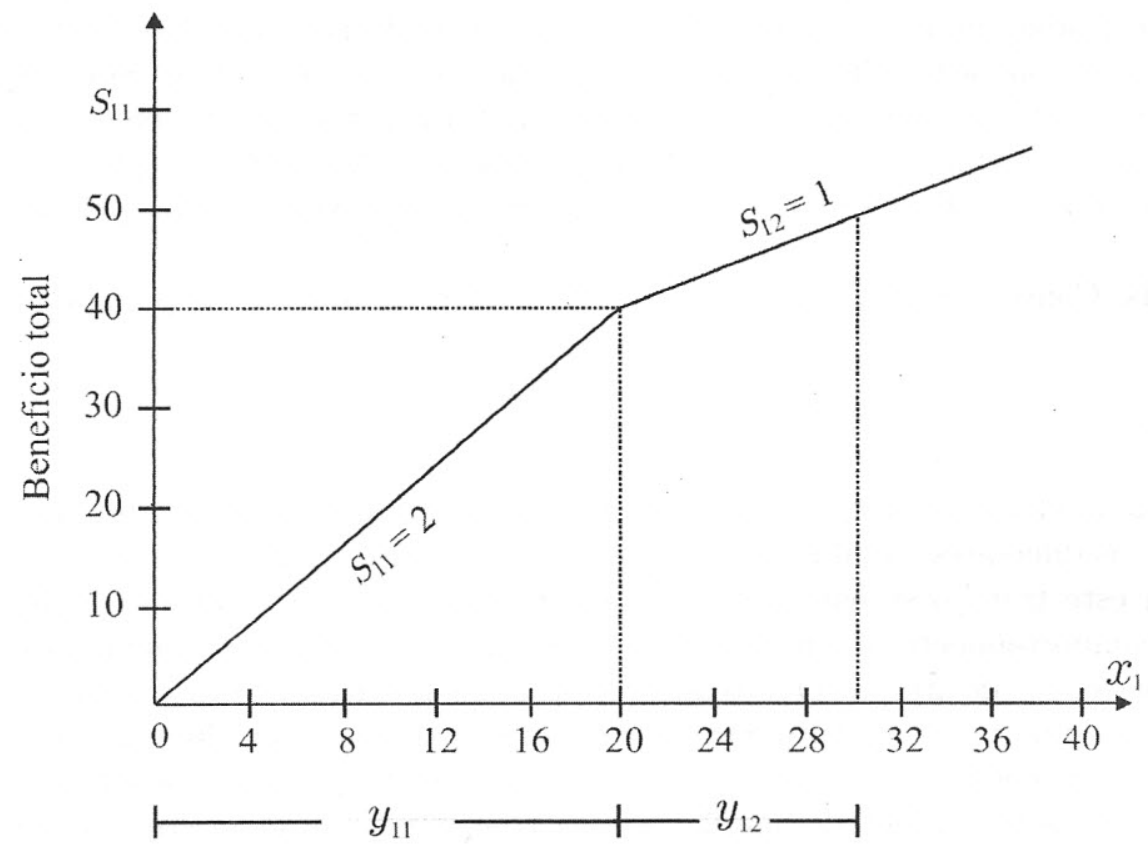

Figura 2

Para este caso, es obvio que la solución óptima factible, es ahora $x_{1}=30$ con $z=2(20)+1(10)=50$ soles. Usaremos este ejemplo para dar una visión del procedimiento.

Como se muestra en la figura 2, podemos tratar el nivel de producción del producto $x_{1}$ sobre las primeras 20 horas que es el tiempo regular como una variable $y_{11}$ y por comodidad el producto producido en las 10 horas adicionales (como sobre tiempo) como otra variable $y_{12}$. De esta manera la cantidad de producto producida en las 20 primeras horas es indistinguible de la que es producida en el sobre tiempo. Por tanto, el beneficio unitario será constante en 2 y 1 soles respectivamente para cada producto sobre su rango permisible. 
Como el beneficio unitario del producto producido en el tiempo regular (de 0 a 20) es mayor que el beneficio unitario del producto producido en el sobre tiempo, estamos seguros que la solución optimal deberá estar fuera de los límites del tiempo regular y antes de los límites del producto producido en el sobretiempo.

Si graficamos las variables $y_{11}$ y $y_{12}$ y sus funciones utilidad total separadamente, vemos que tenemos un típico problema de programación lineal de dos variables.

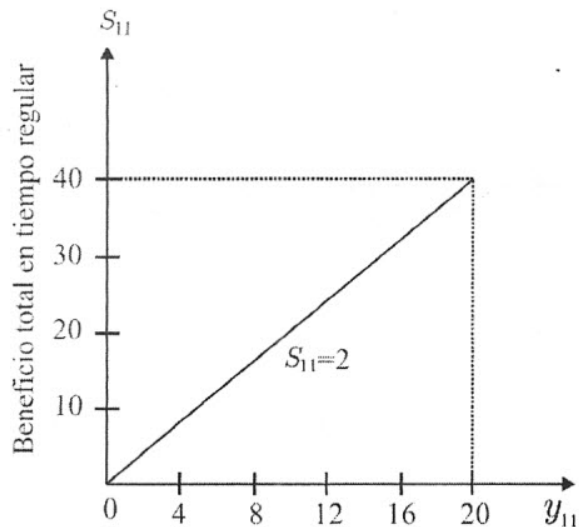

Figura 3

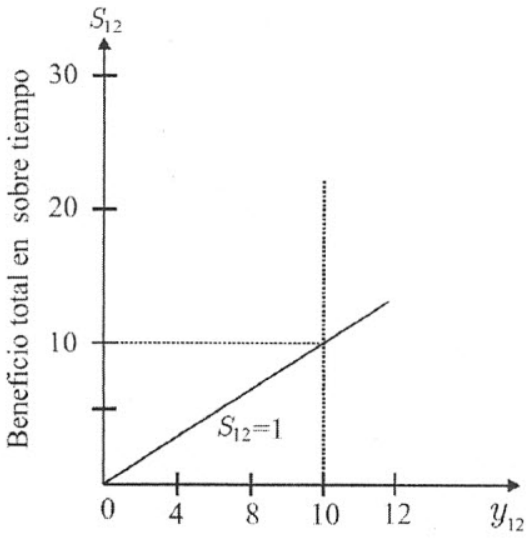

Figura 4

Si introducimos las nuevas variables en la formulación original se obtiene:

$$
\begin{array}{lrl}
\text { Max } & Z & =2 y_{11}+y_{12} \\
\text { s.a. } & \\
& y_{11} \leq 20 \\
y_{12} & \leq 10 \\
y_{11}, y_{12} & \geq 0
\end{array}
$$

La gráfica de la región factible se muestra en la Fig. 5 y también la solución optimal. Por lo tanto,

$$
y_{11}=20, y_{12}=10 \wedge z=2(20)+1(10)=50
$$




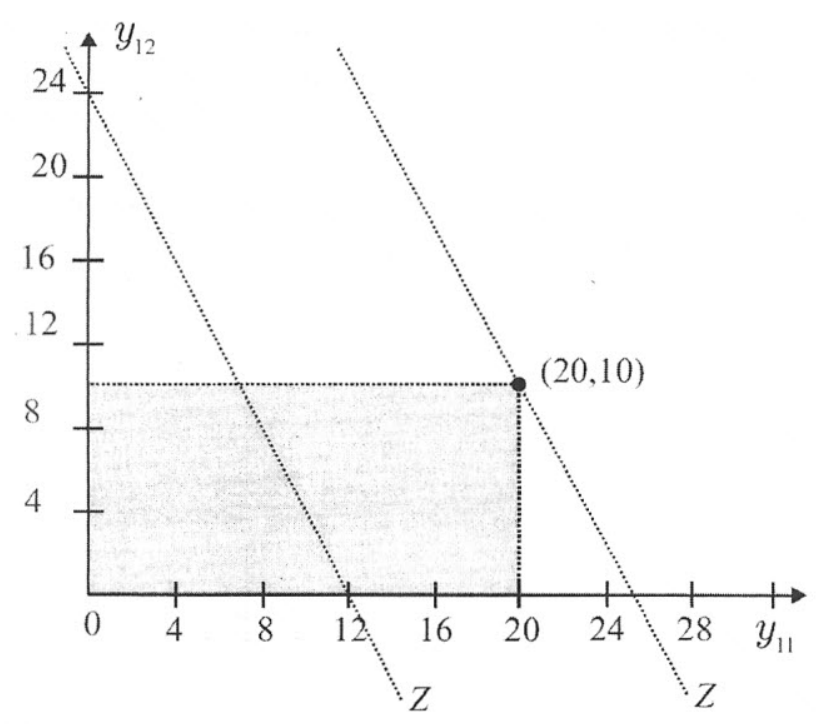

Figura 5

De acuerdo a esto: $x_{1}=y_{11}+y_{12} \Rightarrow x_{1}=20+10=30$

\section{Funciones objetivos separables cóncava y convexas}

\subsection{Función objetivo separable}

Una función objetivo es llamada separable si esta es de la forma siguiente:

$$
f\left(x_{1}, x_{2}, \ldots, x_{n}\right)=\sum_{i=1}^{n} f_{i}\left(x_{i}\right)
$$

donde $f_{j}\left(x_{j}\right)$ es una función que depende solamente de $x_{j}$.

La función objetivo de un problema de Programación Lineal es una función separable donde $f_{1}\left(x_{1}\right)$ es reemplazado por $c_{1} x_{1}, f_{2}\left(x_{2}\right)$ por $c_{2} x_{2}$ y así hasta $c_{n} x_{n}$. Para nuestro problema vamos a considerar que la pendiente de la función utilidad total para el producto $1\left(x_{1}\right)$ sea no constante e igual a $c_{1}$. Análogamente, la pendiente de la función utilidad total para el producto 2 es también no constante e igual a $c_{2}$.

El significado económico de separabilidad de la función objetivo considerado aquí es esencialmente a que: cambios en el nivel de producción de $x_{i}$ no tienen efecto sobre el beneficio unitario de cualquier otro producto. En términos de dos variables, por ejemplo, cambios en $x_{1}$ (en el nivel de salida del producto 1) no tienen efecto sobre la utilidad unitaria asociada con valores de $x_{2}$ (niveles de salida del producto 2) y viceversa. Por otro lado, la total utilidad debido a ambas actividades de producción será la suma de las utilidades generadas por cada actividad de producción.

$$
f\left(x_{1}, x_{2}\right)=f_{1}\left(x_{1}\right)+f_{2}\left(x_{2}\right)
$$

\subsection{Función objetivo cóncava y convexa}

El método de convertir un problema de programación no lineal a un problema de programación lineal es solamente válido si todas las funciones $f_{j}\left(x_{j}\right)$ son cóncavas para el caso de maximización de $f\left(x_{1}, x_{2}, \ldots, x_{n}\right)$ o convexas par el caso de minimización.

Intuitivamente, una función es cóncava si para cualquier par de puntos de la gráfica el segmento que los une esta por debajo de la gráfica.

Las gráficas mostradas en la figura 6, (a) y (b) son funciones cóncavas, mientras que (c) y (d) son convexas.

\subsection{Una formulación general}




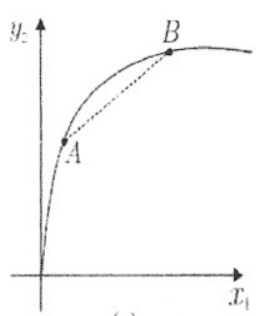

(a)

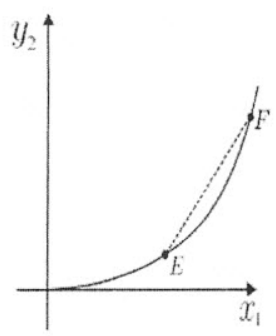

(c)

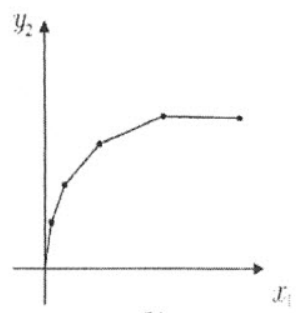

(b)

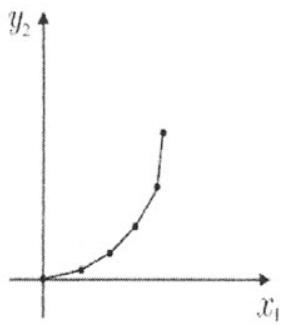

(d)

Figura 6

La principal idea del método es que una función no lineal cóncava o convexa de una sola variable puede ser aproximada tanto como se desee usando pequeños segmentos de recta.

La aproximación será una función lineal continua a trozos. Como ilustración del método mostramos la función cóncava en la figura 7.

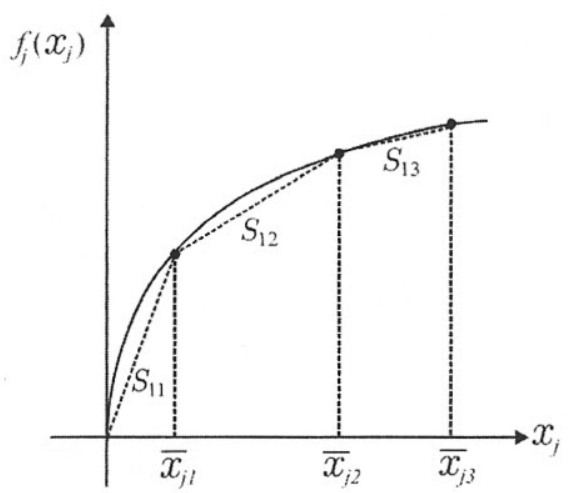

Figura 7

Primero seleccionamos los puntos sobre la gráfica: $f_{j}\left(x_{j}\right)$ las cuales determinan las pendientes de los segmentos que aproximan a $f_{j}\left(x_{j}\right)$. Los valores de $x_{j}$ donde las pendientes cambian son designada por $\bar{x}_{j i}$ como se muestra en la figura 7 . Los puntos de "quiebre" pueden ser pocos o muchos, dependiendo de la exactitud con que se quiere la aproximación. Los valores de $x_{j}$ en el cual el "quiebre" ocurre definen las fronteras de las nuevas variables que serán creadas. Dentro de las fronteras de cada nueva variable la aproximación a $f_{j}\left(x_{j}\right)$ será lineal, como se muestra con líneas "entrecortada" en la figura 7. Así la variable $x_{j}$ podrá ser descompuesta y representada como:

$$
x_{j}=y_{j 1}+y_{j 2}+y_{j 3}+\cdots+y_{j k}
$$

Las condiciones bajo las cuales esas nuevas variables, pueden ser definidas son:

$$
\begin{gathered}
0 \leq y_{j 1} \leq \bar{x}_{j 1}-0 \\
0 \leq y_{j 2} \leq \bar{x}_{j 2}-\bar{x}_{j 1}
\end{gathered}
$$




$$
\begin{gathered}
0 \leq y_{j k} \leq \bar{x}_{j k}-\bar{x}_{j(k-1)} \\
y_{j i} \geq 0
\end{gathered}
$$

La pendiente de cada segmento lineal puede ser representada por $s_{j 1}, s_{j 2}, \ldots, s_{j k}$. Entonces la función $f_{j}\left(x_{j}\right)$ puede ser aproximada por:

$$
f_{j}\left(x_{j}\right)=s_{j 1} y_{j 1}+s_{j 2} y_{j 2}+s_{j 3} y_{j 3}+\cdots+s_{j k} y_{j k}
$$

Luego, sustituyendo la ecuación (2) por cada $x_{j}$ y sustituyendo la ecuación (7) para cada $f_{j}\left(x_{j}\right)$ en la función objetivo obtenemos la formulación del Programa Lineal:

$$
\sum_{i=1}^{n} f_{i}\left(x_{i}\right)=\sum_{j=1}^{n} \sum_{i=1}^{n} s_{j i} y_{j i}
$$

Afortunadamente, las restricciones sobre $y_{j i}$ en las ecuaciones (3), (4), (5) no tienen que ser incluidas en la formulación, porque ellas son tomadas automáticamente con cuidado por la suposición que todas las $f_{j}\left(x_{j}\right)$ son funciones cóncavas, donde las pendientes de los segmentos lineales aparecen en orden decreciente (en magnitud). Por tanto, $y_{j 1}$ será considerado dentro de la solución antes que $y_{j 2}$ e $y_{i 2}$ antes que $y_{i 3}$. y así hasta llegar a $y_{j k}$. Esto es equivalente a:

$$
y_{j k}=0 \quad \text { mientras } \quad y_{j(k-1)} \leq \bar{x}_{j(k-1)}
$$

Por otro lado. para funciones convexas, las pendientes de los segmentos de recta de la función aproximación están en orden creciente (en magnitud).

\section{Ejemplo 2 (Programación cóncava)}

Consideremos una empresa que encara una función de utilidad total cóncava diferente para cada uno de sus dos productos. Para el producto 1 , consideramos $x_{1}$ que representa los niveles de producción de dicho producto, y su beneficio total por este producto es representado por $f_{1}\left(x_{1}\right)$.

Su gráfica y su aproximación lineal es mostrada en la figura 8:

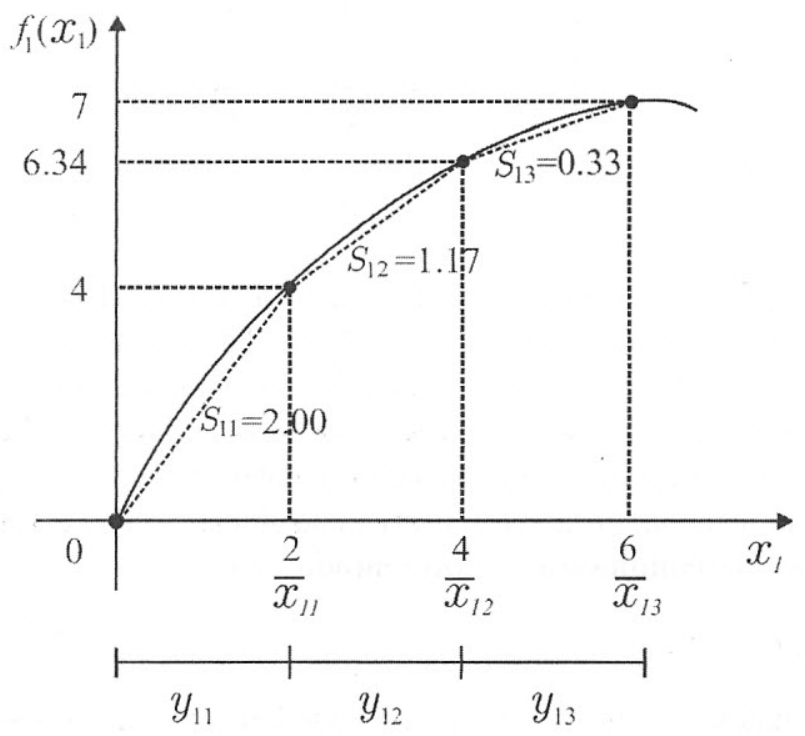

Figura 8

Similarmente, $x_{2}$ representa los niveles de producción del segundo producto y $f_{2}\left(x_{2}\right)$ la apropiada, pero diferente, función utilidad para los niveles de producción del producto 2 . 


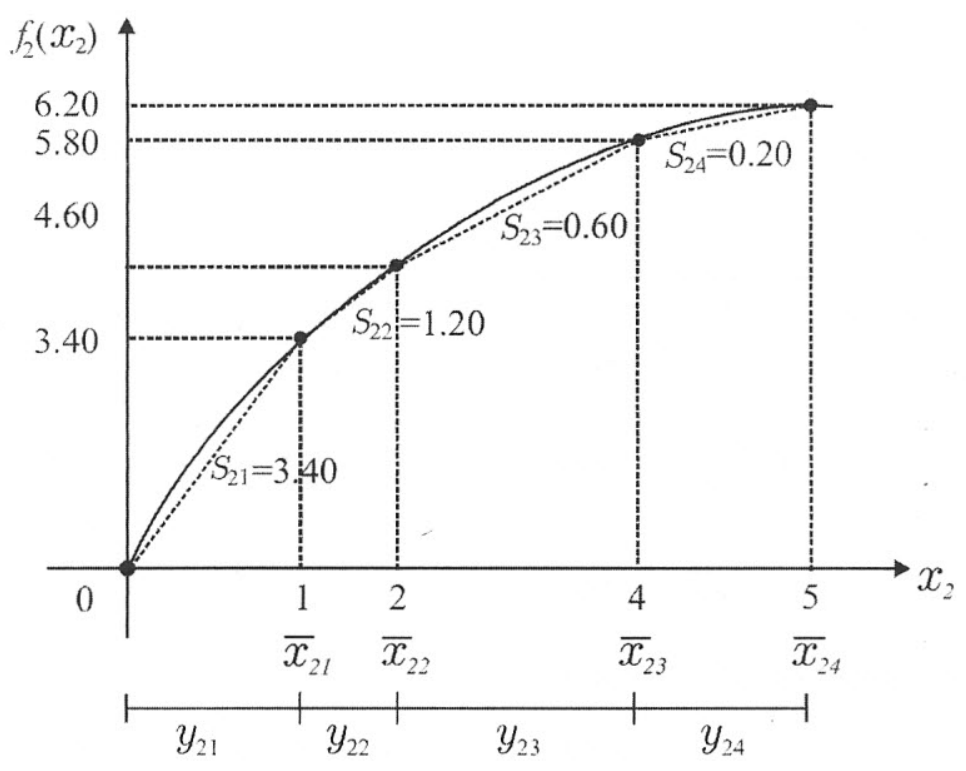

Figura 9

Nuestro objetivo es:

$$
\begin{aligned}
\text { Max } f\left(x_{1}, x_{2}\right) & =f_{1}\left(x_{1}\right)+f_{2}\left(x_{2}\right) \\
\text { s.a. } & \\
x_{1} & \leq 6 \\
x_{2} & \leq 5 \\
x_{1}+2 x_{2} & \leq 12 \\
x_{1}, x_{2} & \geq 0
\end{aligned}
$$

Usando los datos de las figuras 8 y 9, podemos formular el problema de programación lineal que aproxima al problema original.

$\operatorname{Max} Z=2,00 y_{11}+1,17 y_{12}+0,33 y_{13}+3,40 y_{21}+1,20 y_{22}+0,60 y_{23}+0,20 y_{24}$ s.a.

$$
\begin{aligned}
& y_{11}+y_{12}+y_{13} \leq 6 \\
& y_{21}+y_{22}+y_{23}+y_{24} \leq 5 \\
& y_{11}+y_{12}+y_{13}+2\left(y_{21}+y_{22}+y_{23}+y_{24}\right) \leq 12 \\
& y_{11} \leq 2 \\
& y_{12} \quad \leq 2 \\
& y_{13} \leq 2 \\
& y_{21} \leq 1 \\
& y_{22} \quad \leq 1 \\
& y_{23} \quad \leq 2 \\
& \begin{aligned}
y_{24} \quad & \leq 1 \\
y_{i j} & \geq 0
\end{aligned}
\end{aligned}
$$

Resolviendo éste problema usando el método simplex se obtiene:

$$
y^{*}=\left(y_{11}, y_{12}, y_{13}, y_{21}, y_{22}, y_{23}, y_{24}\right)=(2,2,2,1,1,1,0)
$$

De aquí, el nivel de producción del producto 1 es $x_{1}=y_{11}+y_{12}+y_{13}=6$, y el nivel de producción del producto 2, es: $x_{2}=y_{21}+y_{22}+y_{23}+y_{24}=3$.

El beneficio total óptimo alcanzado es:

$$
Z^{*}=12,20
$$

La solución en términos de $x_{1}$ y $x_{2}$ puede ser observada gráficamente en: 


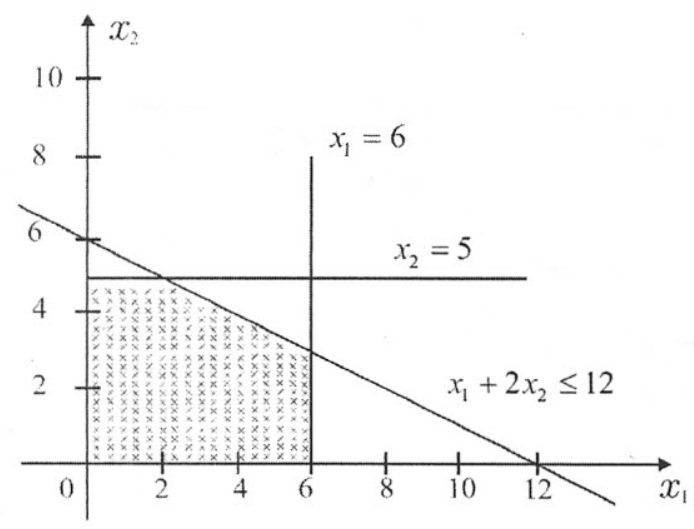

Figura 10

\subsection{Un ejemplo de programación convexa}

Como se mencionó anteriormente, los procedimientos para resolver problemas de minimización son del todo análogos a aquellos para problemas de maximización. La principal diferencia es que en problemas de minimización las funciones costo para cada producto de salida $\left(c_{i}\left(x_{i}\right)\right)$ deben ser convexas.

Así las pendientes de los segmentos lineales que aproximan a las funciones costos están en orden creciente (en magnitud) de izquierda a derecha gráficamente. Esto significa que pequeñas pendientes indican pequeños costos unitarios, para las actividades asociadas a producir hasta cierto límite; si pasan à producir fuera de estos límites significa que los costos cambian a costos mayores.

Como ejemplo consideremos un simple problema de minimización de costos que puede ser resuelto usando el método del transporte: supongamos que se puede producir un solo producto en dos locales ubicados en ciudades diferentes: origen 1 y origen 2 . También existen dos mercados para el producto en lugares distintos: Destino 1, Destino 2. Se tiene además los siguientes datos importantes:

\section{Capacidad de producción \\ Origen 1: 8 unidades \\ Origen 2: 10 unidades \\ Demanda \\ Destino 1: 11 unidades \\ Destino 2: 7 unidades}

Costo unitario para transportar

Origen 1 a Destino 1: 2 soles

Origen 1 a Destino 2: 3 soles

Origen 2 a Destino 1: 5 soles

Origen 2 a Destino 2: 4 soles
Costo unitario de producción

Origen 1: 6 soles por unidad hasta 8 unidades de producción. 12 soles por unidad adicional superior a 8

Origen 2: 5 soles por unidad hasta 10 unidades de producción. 10 soles por unidad adicional superior a 10 .

Podemos construir una tabla 1 para obtener una solución por el método del transporte. En la caja pequeña en la esquina superior izquierda de cada celda tenemos listado primero el costo unitario de transporte por aquella ruta y el segundo el costo de producción para aquel origen representado en aquella fila. Sumando el costo unitario de transporte y el costo unitario de producción para cada celda nos da el coeficiente de costo. Por lo tanto, el costo unitario de producción es el mismo para las celdas de la misma fila pues toda la producción para cada fila posee el mismo origen. 


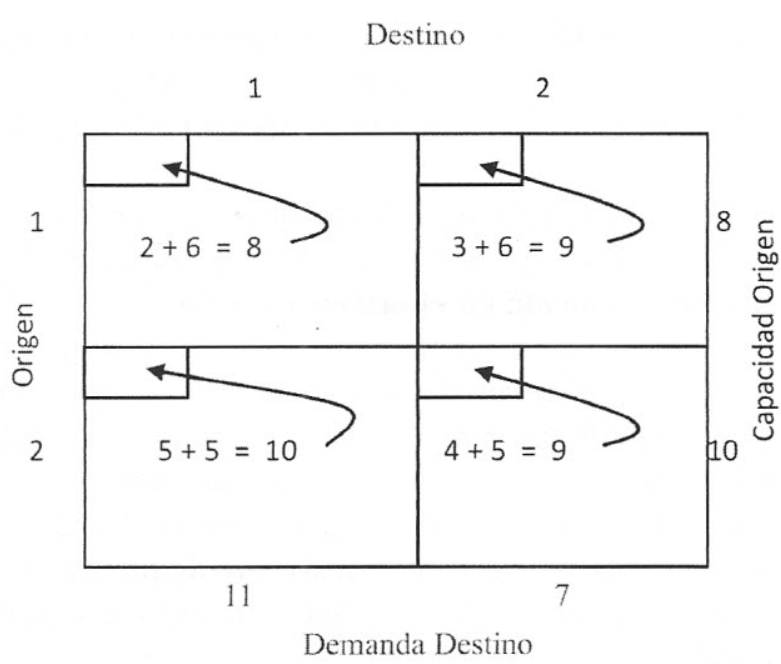

Tabla 1
Destino

1

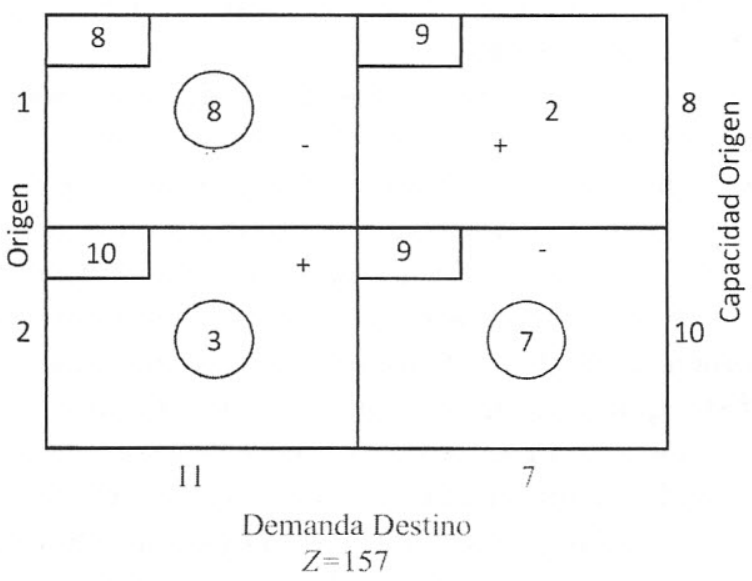

Tabla 2

En la tabla 2, dentro de los círculos tenemos una solución inicial básica factible para nuestro problema. Esta solución básica también es la óptima. Recordemos que podemos identificar una solución básica factible óptima si al evaluar todas las celdas no básicas nos da un valor positivo. En efecto, la evaluación de la única celda no básica $(1,2)$ nos da el valor: $[-8+10-9+9=2]$. Entonces el costo mínimo es $Z=8(8)+9(0)+10(3)+9(7)=157$.

Ahora supongamos que existe incremento en la demanda del destino 2 de 2 unidades, es decir ahora quieren 9 en lugar de 7 . Una interesante pregunta surge, ¿Cuál de los orígenes serviría para satisfacer la demanda del destino 2 ?

Este problema es un problema de programación convexa ahora porque la producción adicional en cada origen incurrirá en un costo unitario de producción mayor al anterior. De los datos de costo de producción se ha esbozado los siguientes gráficos.

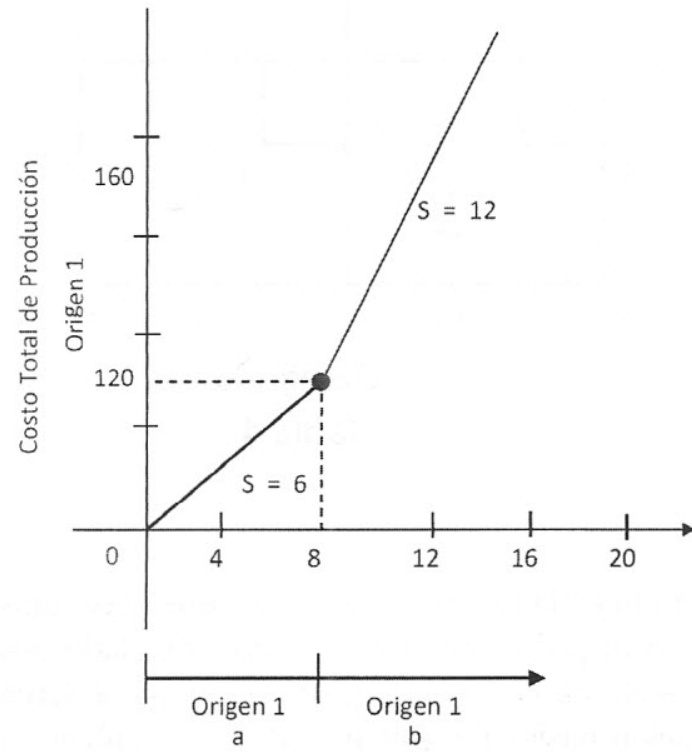

Figura 11

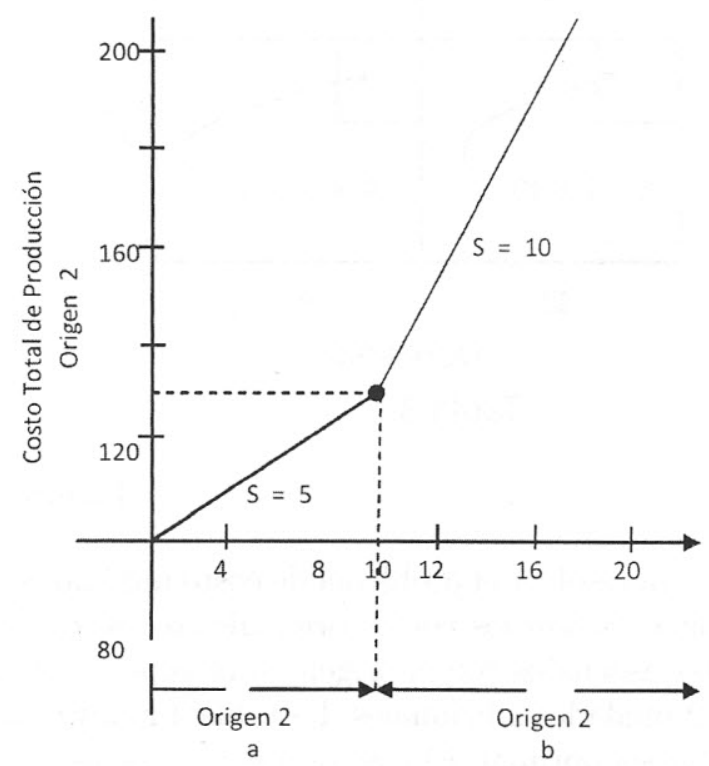

Figura 12

En el origen 1 vemos de la figura 11 que la pendiente de la gráfica del costo de producción es variable, es de 6 soles hasta un nivel de 8 unidades de producción y la pendiente cambia a 12 soles el costo por unidad adicional a 8 unidades. Para el origen 2 ocurre algo similar y el único punto de "quiebre" es en 10 unidades. 
Podemos apreciar de ambos gráficos que el origen 2 incurre en un costo promedio menor que el origen 1. Sin embargo, el origen 1 posee una ventaja en cuanto al costo de transporte, posee en promedio menos que el origen 2. En la mayoría de problemas, el tener numerosos puntos de quiebre en el gráfico es muy apropiado.

Para incorporar los costos de producción asociados a niveles de producción mayores que los iniciales podemos utilizar las ideas propuestas anteriormente. En efecto, podemos tratar lo producido en el origen 1 hasta un límite de 8 unidades como una variable producida en el origen $1_{\mathfrak{a}}$, y para los niveles de producción mayores a 8 unidades en el origen 1 podemos tratarlo como niveles de producción en otro origen $1_{\mathrm{b}}$, es decir como otra nueva variable. Luego, el nivel de producción total del origen 1 para nuestro problema sería la suma de los niveles de producción de los orígenes $1_{\mathrm{a}}$ y $1_{\mathrm{b}}$. Para el caso del origen 2 puede ser tratado de forma análoga y considerando punto de quiebre a 10 unidades.

Esto quiere decir que vamos a considerar dos posibles ubicaciones de las 2 unidades adicionales de producción. Primero determinamos el costo total mínimo (costo de transporte más costo de producción) si toda la producción adicional es producida en el origen 1. Segundo, determinamos el costo total mínimo, suponiendo que toda la producción es producida en el origen 2.

Si las 2 unidades adicionales son producidas en el origen 1, podemos notar de los datos que el costo unitario será de 12 soles por cada unidad adicional.
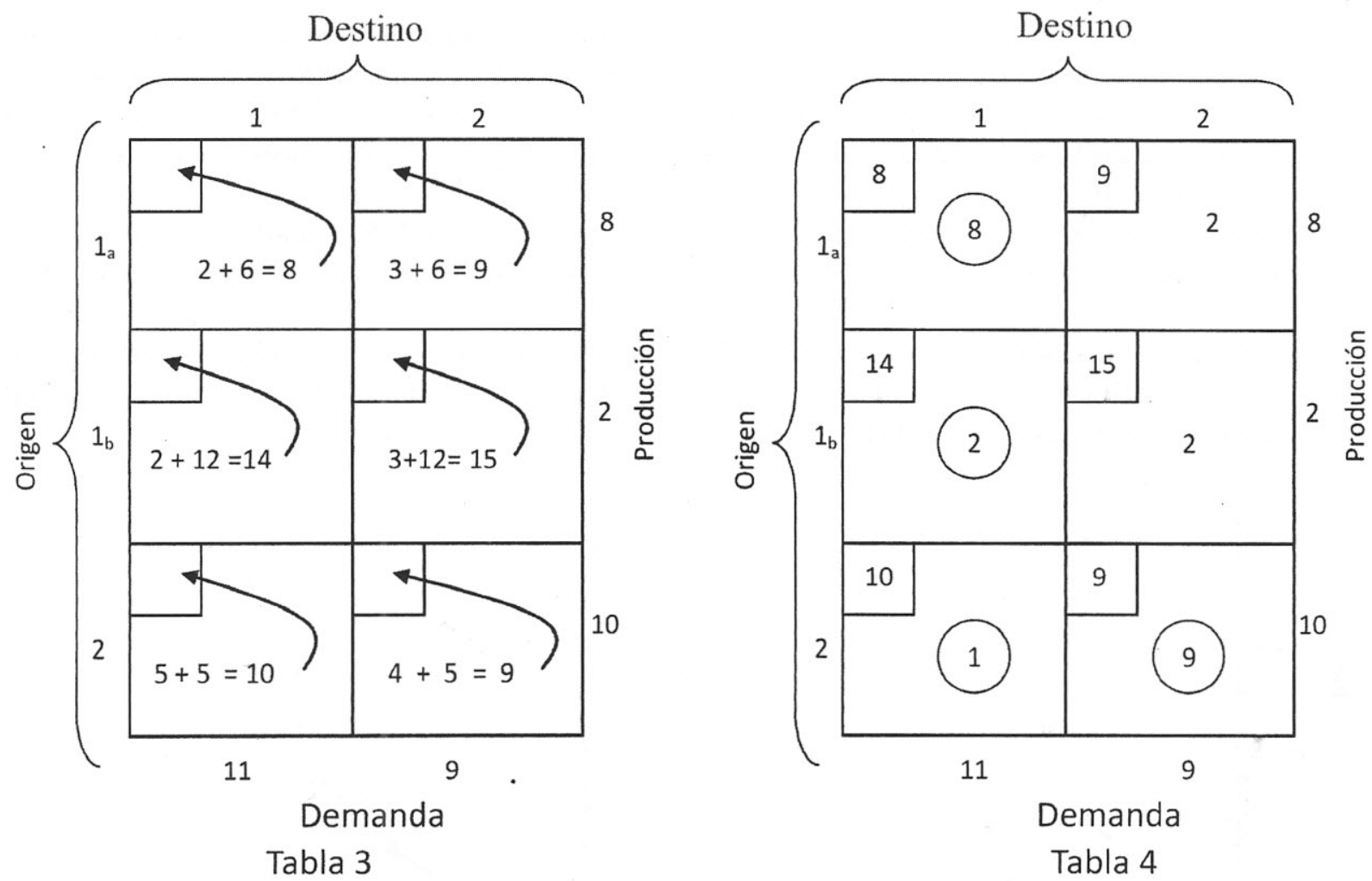

Figura 13

La tabla para resolver el problema de costo mínimo es mostrada en la tabla 3. Los coeficientes asociados cón el origen 1a son los costos originales de producción y transporte del origen 1. Por otro lado, los coeficientes asociados con el origen 1b incorporan el incremento en el costo de producción que acarrea producir 2 unidades adicionales. La tabla 4 muestra la solución básica factible inicial. Esta también es solución básica optimal. El costo total mínimo es

$$
Z=8(8)+9(0)+14(2)+15(0)+10(1)+9(9)=183 \text { soles }
$$

Las 2 unidades adicionales producidas en el origen 1 van hacia el destino 1 y no al destino 2, pues el destino 1 aumenta su demanda. El origen 2 sigue produciendo 10 unidades. Solo que ahora de esta producción 9 unidades van al destino 2 antes iba solo 7 y 1 unidad va al destino 1 (antes solo iba 3). 
La otra alternativa a ser considerada es asumiendo que las 2 unidades de producción adicional son producidas en el origen 2. Esta alternativa es tratada en forma similar al caso anterior. La tabla 5 muestra los apropiados orígenes, destinos, y costos de transporte y producción.
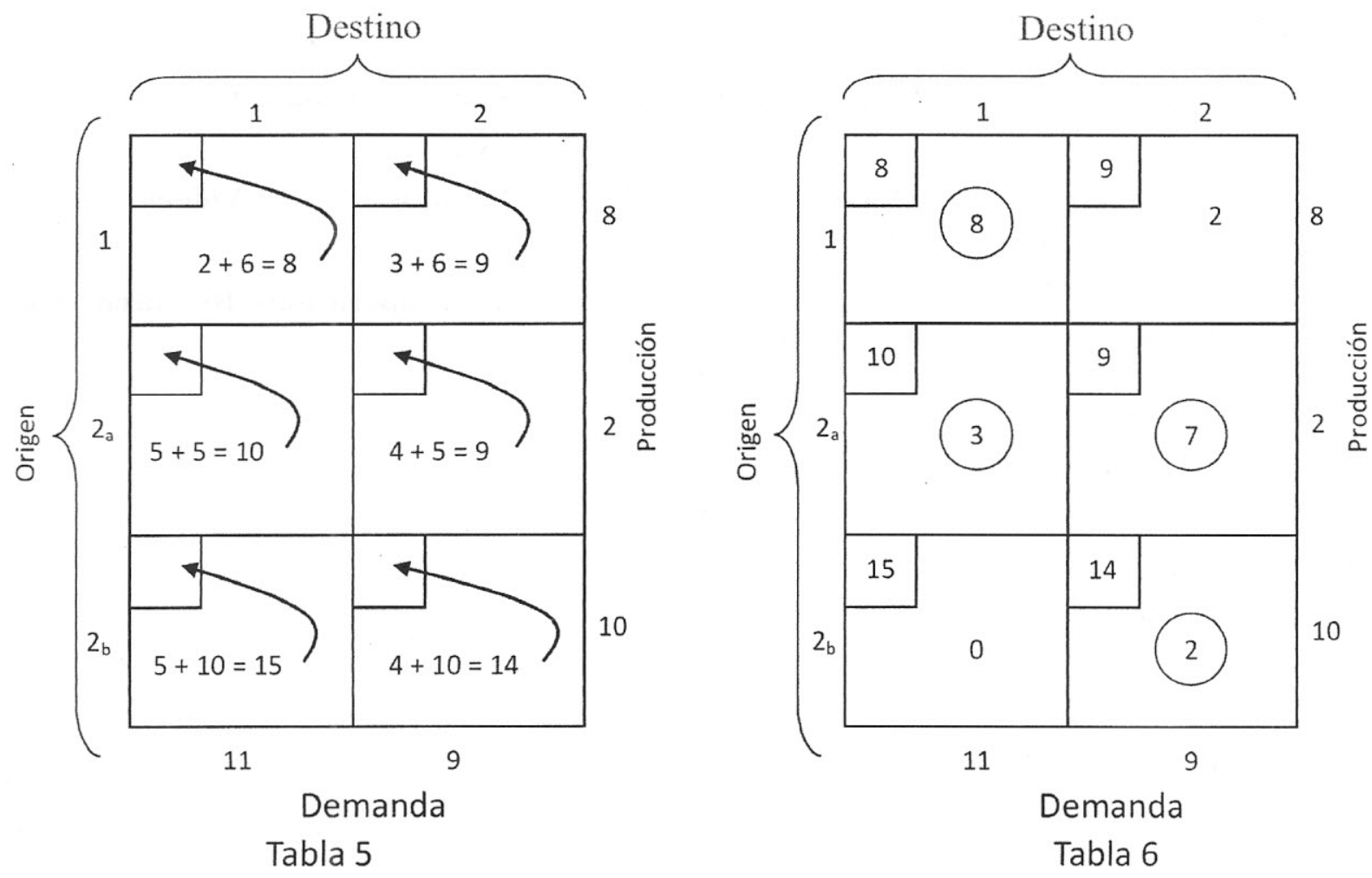

Figura 14

En la tabla 6 se muestra una solución básica factible inicial que también es solución optimal. Al evaluar las celdas no básicas obtenemos un valor de 2 para la celda $(1,2)$ y un valor de cero para la celda $(2 \mathrm{~b}$, 1). La ausencia de cualquier valor negativo indica una solución básica optimal, pero la presencia de un cero en la celda $\left(2_{\mathrm{b}}, 1\right)$ revela la existencia de otra alternativa de solución básica optimal. El costo total mínimo que muestra la tabla 6 es:

$$
Z=8(8)+9(0)+10(3)+9(7)+15(0)+14(2)=185
$$

Ahora observamos que el costo total mínimo para cada una de las alternativas es: si la producción adicional es producida en el origen 1 se genera un costo total de 183 soles. Mientras que si la producción adicional es producida en el origen 2, el costo total que alcanza es 185 soles. Obviamente la primera alternativa es preferida. En un modo similar, los costos de otras alternativas pueden ser estudiadas. El método puede ser extendido a problemas más grandes y más realistas, con varios orígenes y varios destinos. 


\section{REFERENCIAS BIBLIOGRÁFICAS}

[1] Dantzing, George B, Linear Programming and Extensions, Princeton, N. J. Princeton University Press, 1963, chap. 24.

[2] Hadley, G. Nonlinear and Dynamic Programming Reading, Mass, Addison Wesley Publishing Company, Inc., 1964, chap 4.

[3] Hillier, Frederick S., and Gerald J. Lieberman, Introduction to operations Research, San Francisco, Holden - Day, Inc., 1967, pp. 581-592. 